\title{
INFINITE DIMENSIONAL $L$-SPACES DO NOT HAVE PREDUALS OF ALL ORDERS ${ }^{1}$
}

\author{
THOMAS E. ARMSTRONG
}

\begin{abstract}
It is shown that if $E$ is an infinite dimensional Banach space with first dual $E^{\prime}$, second dual $E^{\prime \prime}$, and $n$th dual $E^{[n]}$ and if $E^{[n]}$ is either an $L$ - or $M$-space all duals are either $L$ - or $M$-spaces except possibly $E$ which could be a Lindenstrauss space. If $E$ is an $L$ - or $M$-space there is an integer $n(E)$ so that if $m>n(E)$ there is no Banach space $F$ with $E=F^{[m]}$. The linear isomorphic analogues to these isometric results are also established. In particular if $E$ is an $\mathfrak{L}_{1}$ or $\mathcal{L}_{\infty}$ space there is an integer $\bar{n}(E)$ so that $E$ is not linearly isomorphic to $F^{[m]}$ for any Banach space $F$ when $m>\bar{n}(E)$.
\end{abstract}

Introduction. Consider a sequence $\left\{E_{i}: i=1, \ldots, n\right\}$ of Banach spaces with $E_{j+1}^{\prime}=E_{j}$ for $j=1, \ldots, n-1$. We call $E_{2}$ a predual of $E_{1}$ and $E_{2}$ a pre-bidual of $E_{1}$. In general we say that $E_{j+1}$ is a predual of $E_{1}$ of order $j$ and denote this fact by the equation $E_{j+1}^{(j)}=E_{1}$. Is it possible that there exists, for a given Banach space $E$, an infinite sequence $\left\{E_{j}: j \in N\right\}$ of Banach spaces with $E_{1}=E$ and with $E_{j}$ the dual of $E_{j+1}$ for all $j \in N$ ? If $E$ is reflexive, in particular if $E$ is finite dimensional, this is easily seen to be true taking $E_{j}=E^{\prime}$ if $j$ is even and $E_{j}=E$ if $E$ is odd. James, [6], constructs an example of a nonreflexive separable Banach space $E$ isometric with its bidual. For such a space $E$ the desired sequence $\left\{E_{j}: j \in N\right\}$ is readily produced. If $E$ is required to be an infinite dimensional $L$-space the existence of such a sequence is impossible. This follows from the main result of this paper which asserts the existence of an integer, $n(E)$, for any infinite dimensional $L$-space $E$, such that $E$ has no predual of order $n(E)$. If $E$ is an $L$-space it is known that $E^{[2 n]}$ is also an $L$-space for all $n$. Thus, for all $n$ there are $L$-spaces $E$ with $n(E)$ arbitrarily large. That $n(E)$ may be 0 was established by Gelfand, [4], for $E=L^{1}(\mu)$ where $\mu$ is Lebesgue measure on $[0,1]$ or on $(-\infty, \infty)$, and by Pelcynski, [9], for $\mu \sigma$-finite but not purely atomic. Rosenthal, [13], establishes that when $\Gamma$ is a set whose cardinality, $\operatorname{card}(\Gamma)$, satisfies $\kappa_{0} \leqslant \operatorname{card}(\Gamma)<2^{c}$ then $l^{\infty}(\Gamma)$ does not have a predual of order 3 so that $l^{1}(\Gamma)$, although a dual space, is not a bidual space. Hence, $n\left(l^{1}(\Gamma)\right)=1$. We shall remove the cardinality restriction $\Gamma$ in Proposition 2. Thus, if $\mu$ is a $\sigma$-finite measure and $E=L^{1}(\mu)$ then $n(E)=0$ unless $\mu$ is purely atomic in which case $n(E)=1$.

Received by the editors November 22, 1976 and, in revised form, May 27, 1977 and May 1, 1978.

AMS (MOS) subject classifications (1970). Primary 46B10; Secondary 46A40, 46E30.

$K e y$ words and phrases. $L$-space, $M$-space, Lindenstrauss space, dual, predual, simplex, injective Banach space, topological dimension.

'Supported by National Science Foundation Grant MCS-74-05796-A02. 
This appears to be the extent of precise knowledge of $n(E)$ although we shall give a method for obtaining crude estimates of $n(E)$ for more general $L$-spaces.

1. The preduals of order 2 of an $L$-space. In order to proceed we need to characterize Banach spaces whose $n$th duals are $L$-spaces. It is well known that the dual of an $L$-space ( $M$-space) is an $M$-space with unit ( $L$-space).

If $F$ is an $M$-space which has a predual $E$ then $E$ considered as a subspace of $F^{\prime}$ is unique and is an $L$-space with the induced ordering from the $L$-space $F^{\prime}$. If $E$ is an $L$-space with predual $F$ then $F$ need not be an $M$-space with any ordering. Preduals of $L$-spaces $E$ are called Lindenstrauss spaces. If a Lindenstrauss space, when given the induced ordering from $E^{\prime}$, is an ordered Banach space with $E$ as order-dual it is called a simplex space. The classification of Lindenstrauss spaces is an unwieldy task which has absorbed the research of many persons. The situation for preduals of order $n>1$ could be expected to be even more complicated. Somewhat surprisingly the situation is simplified enormously.

Proposition 1. Let $E$ be a Banach space with $E^{\prime \prime}$ an L-space. $E^{\prime}$ is an $M$-space and $E$ is an L-space.

Proof. Let $B^{\prime}$ and $B^{\prime \prime}$ be the unit balls of $E^{\prime}$ and $E^{\prime \prime}$. There is an extreme point $e$ of $B^{\prime}$ since $B^{\prime}$ is $\sigma\left(E^{\prime}, E\right)$ compact. Lindenstrauss, [7], shows that there is a compact Hausdorff space $X$ and an isometry of $E^{\prime}$ onto a separating subspace $F$ of $e(X)$ such that $e$ is mapped to 1 and such that $F$ has the Riesz separation property with respect to the ordering of $\mathcal{C}(X)$. With the ordering and norm from $\mathcal{C}(X) F$ is order unit normed with order unit $e$. Any such space is isometric and order isomorphic with the space $A(\Delta)$ of continuous affine functions on a Choquet simplex $\Delta$. Thus $E^{\prime}$ is isometric with $A(\Delta)$. Capon shows, [2], that when $A(\Delta)$ is a dual space then $\Delta$ is a Bauer simplex. Thus, $A(\Delta)$ is an $M$-space as is $E^{\prime}$. Since $E$ is the predual of $E^{\prime}$ it is an $L$-space.

Corollary 1-1. Let $E$ be a Banach space with no predual. If, for some integer $n, E^{[n]}$ is an $L$ - or an $M$-space then $E$ is either an $L$-space or a Lindenstrauss space.

Remarks. 1. The following simple category theoretic proof of Proposition 1 is due to K. S. Lau. For terminology and justification of all statements we refer the reader to [14]. We work in the category $\mathrm{Ban}_{1}$ of Banach spaces with linear contractions as morphisms. Any dual $M$-space is injective and all injective objects in this category are $M$-spaces. If $E^{\prime \prime}$ is an $L$-space $E^{\prime}$ is a retract of the injective $E^{\prime \prime \prime}$ hence is itself injective. Thus $E^{\prime}$ is an $M$-space and $E$ is an $L$-space.

2. One might conjecture that if $E$ were a Banach space with $E^{\prime \prime}$ linearly isomorphic to an $L$-space then $E$ would also be linearly isomorphic to an $L$-space. A counterexample appears in Chapter $5 \mathrm{~d}$ of [9]. An example is given 
of a Banach space $E$ not even linearly isomorphic to a complemented subspace of an $L$-space yet with $E^{\prime}$ linearly isomorphic with $l^{\infty}(N)$. Severe restrictions on $E$ are necessary in order that $E^{\prime \prime}$ linearly isomorphic to an $L$-space imply the existence of an $L$-space linearly isomorphic with $E$.

Proposition 2. If $\Gamma$ is an infinite set then $n\left(l^{1}(\Gamma)\right)=1$. In fact $l^{1}(\Gamma)$ is not even linearly isomorphic to a double conjugate space.

Proof. Let Ban be the category of Banach spaces with continuous linear maps as morphisms. Let $E$ be a Banach space with $E^{\prime \prime}$ isomorphic to $l^{1}(\Gamma)$. $E^{\prime \prime \prime}$ is isomorphic to $l^{\infty}(\Gamma)$ which is injective in $\operatorname{Ban}_{1}$ hence $E^{\prime \prime \prime}$ is injective in Ban. Consequently, $E^{\prime}$ is injective in Ban. Corollary 3 of [12], shows that $E^{\prime}$ contains a closed subspace $l$ linearly isomorphic to $l^{\infty}(N)$. Since $l^{\infty}(N)$ is injective in Ban, $l$ is complemented in $E^{\prime}$ hence $E^{\prime \prime}$ contains a complemented subspace linearly isomorphic to $l^{\infty}(N)$ hence to $\Re(\beta N)$. Since $\beta N$ is not scattered there is a nonatomic measure $\mu \in \mathfrak{T}^{+}(\beta N)$, [13]. If $\mathscr{B}$ is a separable $\mu$-nonatomic $\sigma$-algebra of subsets of $N$ the space $L^{1}(\beta N, \Re, \mu)$ is isometric both with $L^{1}\left(\lambda_{1}\right)$ where $\lambda_{1}$ is Lebesgue measure on $[0,1]$ and with the closed subspace $\left\{f \cdot \mu: f \in L^{1}(\beta N, \mathscr{B}, \mu)\right\} \subset \mathscr{T}(\beta N)$. Thus, $l^{1}(\Gamma)$ contains a closed subspace linearly isomorphic to $L^{1}\left(\lambda_{1}\right)$. Since any separable subspace of $l^{1}(\Gamma)$ is in $l^{1}(\Lambda)$ where $\Lambda \subset \Gamma$ has cardinality $\aleph_{0}, l^{1}(N)$ contains a subspace linearly isomorphic with $L^{1}\left(\lambda_{1}\right)$ which is impossible by the remarks on p. 123 of [9].

Proposition 3. If $E$ is a triple conjugate space with $E^{\prime \prime}$ linearly isomorphic to an $L$-space then $E$ is linearly isomorphic to an $L$-space.

Proof. Let $G^{\prime \prime \prime}=E$. $E^{\prime \prime}$ is an $\varrho_{1}$ space so by [9, II.5.8] $G^{\prime}$ and $E$ are $\mathcal{L}_{1}$ spaces whereas $G, G^{\prime \prime}$, and $E^{\prime}$ are $\mathcal{L}_{\infty}$ spaces. From [9, II.5.7] it follows that $G^{\prime \prime}$ is injective in Ban. Haydon, [5] shows that a bidual space injective in Ban is linearly isomorphic to $l^{\infty}(\Gamma)$ for some $\Gamma$. Thus, $E$ is linearly isomorphic to the $L$-space $l^{\infty *}(\Gamma)$. $\square$

REMARKS. 3. If $F$ is an $\mathcal{L}_{\infty}$ space then $F^{\prime \prime}$ is a bidual space injective in Ban. Thus $F^{\prime \prime}$ and all higher even order duals of $F$ are linearly isomorphic to $M$-spaces with the odd order duals linearly isomorphic to $L$-spaces. If $E$ is an $\mathcal{L}_{1}$ space then $E^{[2 n]}$ is linearly isomorphic to an $L$-space and $E^{[2 n-1]}$ is linearly isomorphic to an $M$-space if $n \geqslant 2$.

2. The main result. To establish our result we will show that the dimension of an infinite dimensional $L$-space is strictly less than that of its bidual. As in [13], the dimension of an infinite dimensional Banach space $F, \operatorname{dim}(F)$, is the minimum cardinality of a total subset. Equivalently, $\operatorname{dim}(F)$ is the minimum cardinality of a dense subset, the minimum algebraic dimension of a dense subspace, or the maximum cardinality of a subset, $T$, distinct elements of which are at least a distance 2 apart.

To avoid cumbersome symbolism we shall denote, for cardinal numbers $n$ 
and $m, n^{m}$ by either $\exp (n, m)$ or $\exp ^{1}(n, m)$. If $k \in N$ we define, by induction, $\exp ^{k+1}(n, m)$ to be $\exp \left(n, \exp ^{k}(n, m)\right)$.

If $K$ is a compact Hausdorff space and $\mathfrak{N}(K)=\mathcal{C}^{\prime}(K)$ then $\operatorname{dim}(\mathfrak{N}(K))$ $\geqslant \operatorname{card}(K)$ for the set $\delta(K)=\left\{\delta_{x}, x \in K\right\}$ is a subset such that $\left\|\delta_{x}-\delta_{y}\right\|=$ 2 if $x \neq y$.

If $m$ is a cardinal number we let $\overline{2}^{m}=\{0,1\}^{m}$ and set $\lambda^{m}$ equal to the fair coin toss measure on $\overline{2}^{m}$. If $\lambda_{m}$ is the Lebesgue product measure on $[0,1]^{m}$ it is known that $L^{1}\left(\lambda_{m}\right)$ is isometric with $L^{1}\left(\lambda^{m \cdot \kappa_{0}}\right)$ hence with $L^{1}\left(\lambda^{m}\right)$ when $m$ is infinite. When $m$ is infinite $\operatorname{dim}\left(L^{1}\left(\lambda_{m}\right)\right)$ is known to be $m$ hence $\operatorname{dim}\left(L^{1}\left(\lambda^{m}\right)\right)$ $=m$.

If $\left\{E_{\alpha}\right\}$ is a family of Banach spaces, the $l^{1}$-direct sum, $\left(\Sigma_{\alpha} E_{\alpha}\right)_{1}$, is the set of all $e=\left(e_{\alpha}\right) \in \Pi_{\alpha} E_{\alpha}$ such that $\|e\|_{1}=\Sigma_{\alpha}\left\|e_{\alpha}\right\|<\infty$. The $l^{\infty}$-direct sum, $\left(\Sigma_{\alpha} E_{\alpha}\right)_{\infty}$, is the set of all $e=\left(e_{\alpha}\right) \in \Pi_{\alpha} E_{\alpha}$ such that $\|e\|_{\infty}=\sup _{\alpha}\left\|e_{\alpha}\right\|<\infty$. $\left(\Sigma_{\alpha} E_{\alpha}\right)_{1}$, with the norm \|\|$_{1}$, is a Banach space whose dual is $\left(\Sigma_{\alpha} E_{\alpha}^{\prime}\right)_{\infty}$ with the norm \|\|$_{\infty}$. The dimension of $\left(\Sigma_{\alpha} E_{\alpha}\right)_{1}$ is $\Sigma_{\alpha} \operatorname{dim}\left(E_{\alpha}\right)$ where $\operatorname{dim}\left(E_{\alpha}\right)$ is the algebraic dimension of $E_{\alpha}$ if $E_{\alpha}$ is finite dimensional, for, if $T_{a}$ is total in $E_{\alpha}$ for all $\alpha$ then $\cup_{\alpha} T_{\alpha}$ is total in $\left(\Sigma_{\alpha} E_{\alpha}\right)_{1}$. If $\Gamma$ is a set with cardinality $m$ then $\operatorname{dim}\left(l^{1}(\Gamma)\right)=m$ for $l^{1}(\Gamma)$ is an $l^{1}$-direct sum of one dimensional spaces.

If $G$ is a Banach space with $m=\operatorname{dim}(F) \geqslant \aleph_{0}$ and $T$ is a total subset of $G$ then $G^{\prime}$ maybe considered to be a subset of $(-\infty, \infty)^{T}$. Since

$$
\operatorname{card}\left((-\infty, \infty)^{T}\right)=\exp \left(\exp \left(2, \aleph_{0}\right), m\right)=\exp (2, m)
$$

when $m \geqslant \aleph_{0} \operatorname{dim}\left(G^{\prime}\right) \leqslant \operatorname{card}\left(G^{\prime}\right) \leqslant \exp (2, m)$. Similarly, $\operatorname{dim}\left(G^{\prime \prime}\right) \leqslant$ $\exp ^{2}(2, m)$ and, in general, $\operatorname{dim}\left(G^{[k]}\right) \leqslant \exp ^{k}(2, m)$ for all $k \in N$.

If $m \geqslant \aleph_{0}$ is the cardinal of a set $\Gamma$ then $l^{\infty}(\Gamma)$ is isometric with $\mathcal{C}(\beta \Gamma)$ hence

$$
\operatorname{dim}\left(l^{\infty^{\prime}}(\Gamma)\right)=\operatorname{dim}(\mathscr{T}(\beta \Gamma)) \geqslant \operatorname{card}(\beta \Gamma)=\exp ^{2}(2, m) .
$$

Since $l^{\infty^{\prime}}(\Gamma)=\left[l^{1}(\Gamma)\right]^{\prime \prime}, \operatorname{dim}\left(l^{\infty^{\prime}}(\Gamma)\right)=\exp ^{2}(2, m)$.

If $m \geqslant \kappa_{0}$ then $\mathcal{C}\left(\overline{2}^{m}\right)$ is isometric to a subspace of $L^{\infty}\left(\lambda^{m}\right)$ hence $\mathscr{T}\left(\overline{2}^{m}\right)$ is isometric to a quotient space of $L^{\infty^{\prime}}\left(\lambda^{m}\right)$. Thus $\operatorname{dim}\left(L^{\infty^{\prime}}\left(\lambda^{m}\right)\right) \geqslant$ $\operatorname{dim}\left(\mathfrak{T}\left(\overline{2}^{m}\right)\right) \geqslant \operatorname{card}\left(\overline{2}^{m}\right)=2^{m}$

Proposition 4. If $E$ is an L-space with $m=\operatorname{dim}(E)>\aleph_{0}$ then $\exp (2$, $m) \leqslant \operatorname{dim}\left(E^{\prime \prime}\right) \leqslant \exp ^{2}(2, m)$.

Proof. It is only necessary to show that $\exp (2, m) \leqslant \operatorname{dim}\left(E^{\prime \prime}\right)$.

We may assume by the Kakutani-Maharam Representation Theorem, [14], that $E=\left[l^{1}(\Gamma)+\sum_{\alpha \in \Lambda} L^{1}\left(\lambda^{m_{\alpha}}\right)\right]_{1}$ with $\Gamma$ and $\Lambda$ sets and $m_{\alpha}>\aleph_{0}$ for $\alpha \in \Lambda$.

Case 1. $m=\operatorname{card}(\Gamma)$. $E$ contains $l^{1}(\Gamma)$ isometrically hence $E^{\prime \prime}$ contains $l^{\infty}(\Gamma)$ isometrically. Thus

$$
\operatorname{dim}\left(E^{\prime \prime}\right) \geqslant \operatorname{dim}\left(l^{\infty^{\prime}}(\Gamma)\right)=\exp ^{2}(2, m)>\exp (2, m) .
$$

Case 2. $m=m_{\alpha}$ for some $\alpha \in \Lambda$. E contains $L^{1}\left(\lambda^{m}\right)$ isometrically hence

$$
\operatorname{dim}\left(E^{\prime \prime}\right) \geqslant \operatorname{dim}\left(L^{\infty^{\prime}}\left(\lambda^{m}\right)\right) \geqslant \exp (2, m)
$$


Case 3. $m>\operatorname{card}(\Gamma)$ and $m>m_{\alpha}$ if $\alpha \in \Lambda$. In this case

$$
m=\sum_{\alpha \in \Lambda} m_{\alpha}=\sup _{\alpha \in \Lambda} m_{\alpha} \text {. }
$$

Regard each $m_{\alpha}$ as the first ordinal of cardinal $m_{\alpha}$. Well order $\Lambda$ so that $\left\{m_{\alpha}: \alpha \in \Lambda\right\}$ is nondecreasing along $\Lambda$. If the ordinals $m$ and $m_{\alpha}$ are regarded as sets then $m$ is the increasing union $\cup_{\alpha \in \Lambda} m_{\alpha}$. Let $\pi_{\alpha}: \overline{2}^{m} \rightarrow \overline{2}^{m_{\alpha}}$ be the natural projection restricting a function on the set $m$ to the smaller set $m_{\alpha}$. Let $\mathscr{F}_{\alpha}$ be the Borel sets in $\overline{2}^{m_{\alpha}}$, let $\mathscr{F}$ be the Borel sets in $\overline{2}^{m}$ and let $\mathcal{G}_{\alpha}=\pi_{\alpha}^{-1}\left(\mathscr{F}_{\alpha}\right)$ for $\alpha \in \Lambda$. The set $\left\{\mathcal{G}_{\alpha} \alpha \in \Lambda\right\}$ is an increasing family of $\sigma$-algebras. The inverse image of the measure $\lambda^{m_{\alpha}}$ on $\mathcal{G}_{\alpha}$ under $\pi_{\alpha}$ is the restriction of $\lambda^{m}$ to $\mathcal{G}_{\alpha}$. Equivalently, $\lambda^{m_{\alpha}}$ is the image of $\lambda^{m}$ on $2^{m_{\alpha}}$ under $\pi_{\alpha}$. The map $j_{\alpha}$ : $f \rightarrow f \circ \pi_{\alpha}$ yields an isometric embedding of $L^{1}\left(\lambda^{m_{\alpha}}\right)$ into $L^{1}\left(\lambda^{m}\right)$ with range $L^{1}\left(\overline{2}^{m}, \mathcal{G}_{\alpha}, \lambda^{m}\right)$. If $f=\left(f_{\alpha}\right) \in\left[\Sigma_{\alpha \in \Lambda} L^{1}\left(\lambda^{m_{\alpha}}\right)\right]_{1}$ define $j(f)$ as $\Sigma_{\alpha \in \Lambda} j_{\alpha}\left(f_{\alpha}\right)$. The map $j$ is of norm 1 from $\left[\sum_{\alpha \in \Lambda} L^{1}\left(\lambda^{m_{\alpha}}\right)\right]_{1}$ into $L^{1}\left(\lambda^{m}\right)$. If $g \in L^{1}\left(\lambda^{m}\right)$ and $\alpha \in \Lambda$ then $E\left(g \mid \mathcal{G}_{\alpha}\right)$ is in the range of $j_{\alpha}$ hence is in that of $j$ and $\left\|E\left(g \mid \mathcal{G}_{\alpha}\right)\right\|$ $<\|g\|$. By the Martingale Convergence Theorem,

$$
\lim _{\alpha \in \Lambda} E\left(g \mid \mathcal{G}_{\alpha}\right)=g
$$

in $L^{1}\left(\lambda^{m}\right)$. This shows that the image of the unit ball in $\left[\sum_{\alpha \in \Lambda} L^{1}\left(\lambda^{m_{\alpha}}\right)\right]_{1}$ is dense in that of $L^{1}\left(\lambda^{m}\right)$. From this it follows that $j$ is a surjection and that $L^{1}\left(\lambda^{m}\right)$ is isometric to the quotient of $\sum_{\alpha \in \Lambda} L^{1}\left(\lambda^{m_{\alpha}}\right)$ by $\operatorname{ker}(j)$ hence that $L^{1}\left(\lambda^{m}\right)$ is isometric to a quotient space of $E$. Thus, $L^{\infty^{\prime}}\left(\lambda^{m}\right)$ is isometric with a quotient space of $E^{\prime \prime}$ and $\operatorname{dim}\left(E^{\prime \prime}\right) \geqslant \operatorname{dim}\left(L^{\infty^{\prime}}\left(\lambda^{m}\right)\right) \geqslant \exp (2, m)$.

Corollary 4-1. Let $E$ be an L-space with $m=\operatorname{dim}(E) \geqslant \aleph_{0}$. If $k \in N$ then $\exp ^{k}(2, m) \leqslant \operatorname{dim}\left(E^{[2 k]}\right) \leqslant \exp ^{2 k}(2, m)$.

Corollary 4-2. If $E$ is an L-space with $\aleph_{0} \leqslant \operatorname{dim}(E) \leqslant \exp ^{k}\left(2, \aleph_{0}\right)$ for some $k \in N$ then $n(E)<2 k$.

Corollary 4-3. If $E$ is an L-space with $\operatorname{dim}(E) \geqslant \exp ^{k}\left(2, \aleph_{0}\right)$ for all $k \in N$ then $E$ has no separable predual of any order.

COROLlary 4-4. If $F$ is an infinite dimensional Banach space and $k \in N$ with $F^{[k]}$ linearly isomorphic to an L-space then $F^{[n]}$ and $F^{[m]}$ are not linearly isomorphic for any $n \neq m$ in $N$.

This last corollary is very well known.

Corollary 4-5. If $E$ is an infinite dimensional L-space it is nonreflexive.

Proposition 5. If $E$ is an infinite dimensional $\mathcal{L}_{1}$ (or $\mathcal{L}_{\infty}$ space) there is an integer $\bar{n}(E)<\infty$ so that if $F$ is linearly isomorphic to $E$ then $n(F) \leqslant \bar{n}(E)$.

Proof. Let $C$ be the set of cardinal numbers $m$ so that there is an $L$-space $F$ with $E$ linearly isomorphic to $F^{[2 n]}$ for some integer $n$ and with $m=$ $\operatorname{dim}(F)$. Let $M=\min (C)$. Let $k_{0}$ be an integer and let $F_{0}$ be an $L$-space with $\operatorname{dim}\left(F_{0}\right)=M$ and with $F_{0}^{\left[2 k_{0}\right]}$ linearly isomorphic to $E$. If $G$ is any $L$-space 
and $j$ is an integer with $G^{[2 j]}$ linearly isomorphic with $E$, Corollary 4-1 and the fact that $\operatorname{dim}(G) \geqslant M$ implies that

$$
\exp ^{j}(2, M) \leqslant \operatorname{dim}\left(G^{[2 j]}\right)=\operatorname{dim}(E)=\operatorname{dim}\left(F_{0}^{\left[2 k_{0}\right]}\right) \leqslant \exp ^{2 k_{0}}(2, M) .
$$

Thus $j \leqslant 2 k_{0}$.

If $E$ is not isomorphic with a fourth conjugate space then $\bar{n}(E) \leqslant 3$. Otherwise, if $E$ is linearly isomorphic to $F^{[2 k]}$ with $k \geqslant 2$ Proposition 3 and Remarks 3 show that $F^{[4]}$ is isomorphic to an $L$-space $G$. Thus $k-2 \leqslant 2 k_{0}$. Allowing for the possibility that $F$ is a dual space it follows that $\bar{n}(E)<2 k_{0}$ +3 .

Corollary 5-1. If $E$ is an L-space (or M-space) then $n(E)<\infty$.

ACKNOwledgements. We wish to thank C. McCarthy for several helpful conversations.

We especially wish to thank Haskell Rosenthal who suggested the extension of the isometric results of this paper to linear isomorphism results and who provided most of the details of this extension.

\section{REFERENCES}

1. E. M. Alfsen, Convex compact sets and boundary integrals, Springer, New York, 1971.

2. M. Capon, Etude des espaces $A(K)$ qui sont des duaux, Math. Scand. 32 (1973), 225-241.

3. A. J. Ellis, The duality of partially ordered normed linear spaces, J. London Math. Soc. 39 (1964), 730-744.

4. I. M. Gelfand, Abstrakte Funktionen und lineare Operatoren, Mat. Sb. 46 (1938), 235-286.

5. R. Haydon, On dual $L^{1}$-spaces and injective bidual Banach spaces (unpublished).

6. R. C. James, A non-reflexive Banach space isometric with its second conjugate, Proc. Nat. Acad. Sci. U.S.A. 37 (1951), $174-177$.

7. J. Lindenstrauss, Extensions of compact operators, Mem. Amer. Math. Soc. No. 48, 1964.

8. _ A remark on $\mathfrak{L}_{1}$-spaces, Israel J. Math. 8 (1970), 80-82.

9. J. Lindenstrauss and L. Tzafiri, Classical Banach spaces, Springer-Verlag, New York, 1973.

10. A. Pelczynski, On Banach spaces containing $L^{1}(\mu)$, Studia Math. 30 (1968), 231-246.

11. On the isomorphism of the spaces $m$ and $M$, Bull. Acad. Polon. Sci. Sér. Sci. Math. Astronom. Phys. 6 (1958), 695-696.

12. H. P. Rosenthal, On complemented and quasi-complemented subspaces of quotients of $C(S)$ for Stonian S, Proc. Nat. Acad. Sci. U.S.A. 60 (1968), 1165-1169.

13. , On injective Banach spaces and the spaces $L^{\infty}(\mu)$ for finite measures $\mu$, Acta Math. 124 (1970), 205-248.

14. Z. Semadeni, Banach spaces of continuous functions. I, PWN-Polish Scientific, Warsaw, 1971.

School of Mathematics, University of Minnesota, Minneapolis, Minnesota 55455 